\title{
Acquiring, archiving, analyzing and exchanging seismic data in real time at the Seismological Research Center of the OGS in Italy
}

\author{
Pier Luigi Bragato ${ }^{1}$, Paolo Di Bartolomeo ${ }^{1}$, Damiano Pesaresi ${ }^{1,2, \star}$, \\ Milton Percy Plasencia Linares ${ }^{1}$, Angela Saraò ${ }^{1}$ \\ ${ }^{1}$ Istituto Nazionale di Oceanografia e di Geofisica Sperimentale - OGS, Udine, Italy
${ }^{2}$ Istituto Nazionale di Geofisica e Vulcanologia - INGV, Rome, Italy
}

Article history

Received May 5, 2010; accepted January 25, 2011.

Subject classification:

Surveys, measurements and monitoring, Instruments and techniques, Seismic risk, Data processing, Seismological data.

\section{ABSTRACT}

After the 1976 Friuli earthquake $(\mathrm{Ms}=6.5)$ in north-eastern Italy that caused about 1,000 casualties and widespread destruction in the Friuli area, the Italian government established the Centro di Ricerche Sismologiche (CRS). This is now a department of the Istituto Nazionale di Oceanografia e di Geofisica Sperimentale (OGS), and it is specifically devoted to the monitoring of the seismicity of north-eastern Italy. Since its inception, the North-East Italy Seismic Network has grown enormously. Currently, it consists of 14 broad-band and 20 short-period seismic stations, all of which are telemetered to and acquired in real time at the OGS-CRS data center in Udine. Data exchange agreements in place with other Italian, Slovenian, Austrian and Swiss seismological institutes lead to a total number of 94 seismic stations acquired in real time, which confirms that the OGS is the reference institute for seismic monitoring of north-eastern Italy. Since 2002, CRS has been using the Antelope software suite as the main tool for collecting, analyzing, archiving and exchanging seismic data. SeisComP is also used as a real-time data exchange server tool. A customized webaccessible server is used to manually relocate earthquakes, and automatic procedures have been set-up for moment-tensor determination, shaking-map computation, web publishing of earthquake parametric data, waveform drumplots, state-of-health parameters, and quality checks of the station by spectra analysis. Scripts for email/SMS/fax alerting to public institutions have also been customized. Recently, a real-time seismology website was designed and set-up (http://rts.crs.inogs.it/).

\section{Introduction}

The seismicity of north-eastern Italy (Figure 1) demarcates the boundary between the Adria microplate and the Eurasian plate. The present state of stress is a consequence of the progressive motion of the Adria microplate and its anti-clockwise rotation with respect to the Eurasian plate. The seismotectonic characteristics of the region are not homogeneous, and the contemporary seismic deformation pattern is quite complex, as it is the result of the superimposition of several distinct strain fields that are related to different Alpine phases [e.g. Bressan et al. 2003]. The last severe earthquake was the 1976 Friuli earthquake, Ms $=6.5$, which caused lot of damage and resulted in hundreds of casualties [Carulli and Slejko 2005, and references therein].

The Centro di Ricerche Sismologiche (CRS; Seismological Research Center) of the Istituto Nazionale di Oceanografia e di Geofisica Sperimentale (OGS) was established by the Italian government after this destructive 1976 Friuli earthquake. It manages an integrated seismic network (Figure 2) that is designed to monitor regional seismic activity of north-eastern Italy and its surroundings. The North-East Italy Seismic Network includes 14 digital broad-band seismometers and 20 short-period stations, all of which are telemetered to and acquired in real time at the OGS-CRS data center in Udine. Waveforms and parametric data are exchanged for a total of 94 seismic stations acquired in real time, with the local Civil Defence agencies in Italy (Regione Autonoma Friuli-Venezia Giulia, Regione Veneto, Provincia Autonoma di Trento, Provincia Autonoma di Bolzano), the Istituto Nazionale di Geofisica e Vulcanologia (INGV), the University of Trieste, the Zentralanstalt für Meteorologie und Geodynamik (ZAMG) in Vienna, the Agencija Republike Slovenije za Okolje (ARSO) in Ljubljana, the Schweizerischer Erdbebendienst (SED) of the Eidgenössische Technische Hochschule (ETH) in Zurich. This wide virtual network supports emergency management and seismological studies in north-eastern Italy, as well as in the whole Alps-Dinarides junction zone.

\section{Seismological instrumentation and data transmission}

The seismological stations managed by the OGS are deployed in the Friuli-Venezia Giulia and Veneto regions (Figure 2). The 14 broad-band stations are equipped with 24bit Quanterra data loggers (Q330, Q4120 and Q680) and a variety of seismometers with natural periods between $40 \mathrm{~s}$ 


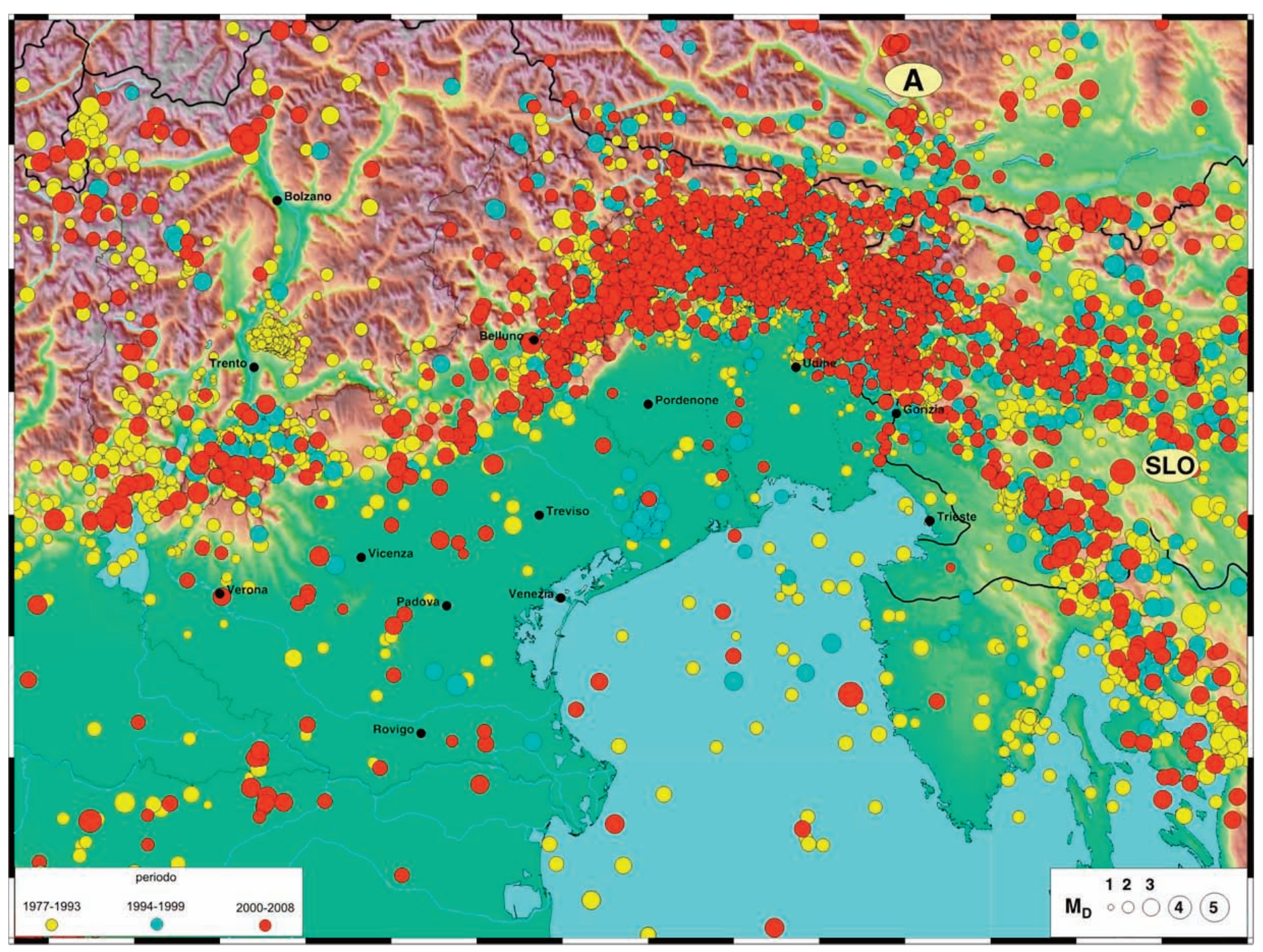

Figure 1. Seismicity of north-eastern Italy recorded at the OGS-CRS between 1977 and 2008 (courtesy of S. Urban). The 1976 Friuli earthquake, Ms = 6.5, is the largest recorded to date in the area.

and $360 \mathrm{~s}$ (Streckeisen STS-1 and STS-2, Nanometrics Trillium-120 and Trillium-40). Most of the stations also include an accelerometer (Kinemetrics EpiSensor ES-T or Guralp CMG-5T). The 20 short-period stations have a 1-Hz Lennartz LE-3Dlite seismometer and a Lennartz MARS-88 data logger. Broad-band stations are acquired continuously, while the short-period stations transmit event data detected on the basis of a short-term averaging/long-term averaging (STA/LTA) triggering algorithm. The overall transmission system is heterogeneous and includes UHF and spreadspectrum radio links, as well as some General Packet Radio Service (GPRS) connections. For one station, we are at present experimenting with a redundant satellite/GPRS double connection. The spread-spectrum communication is based on a complex infrastructure (a backbone that includes a number of repeaters transmitting at $6 \mathrm{Mbps}$ ) that is entirely developed and managed by the OGS-CRS.

\section{Data acquisition, management and sharing}

In 2002, the Antelope software suite, from Boulder RealTime Technologies (BRTT), was chosen as the common basis for analyzing, archiving and exchanging seismic data with the north-eastern Italy border countries (Austria and Slovenia) in the framework of the EU Interreg IIIA project: "Transnational seismological networks in the south-eastern Alps».

Figure 3 shows a flow chart that illustrates the Antelope architecture that has been customized for the OGS-CRS needs. The software is used as the data acquisition tool by the seismic stations. Broad-band data are collected through direct IP connections to the data loggers using the programs of the suite (e.g., «q3302orb» for the Quanterra Q330). Shortperiod data are gathered by a proprietary acquisition system, by Lennartz, and then forwarded to Antelope using the program «m882orb", which was developed at the OGS.

The main OGS Antelope server is running at the CRS in Udine on a two-node SUN-Fire-V240 cluster: the main module takes care of the data acquisition, automatic location determination, data archiving and exchange, and the alert system via Short Message Service (SMS), email, fax and web. Another Linux workstation running Antelope at the OGSCRS headquarters in Udine is used for post-processing, including shake maps and manual relocation determinations. 


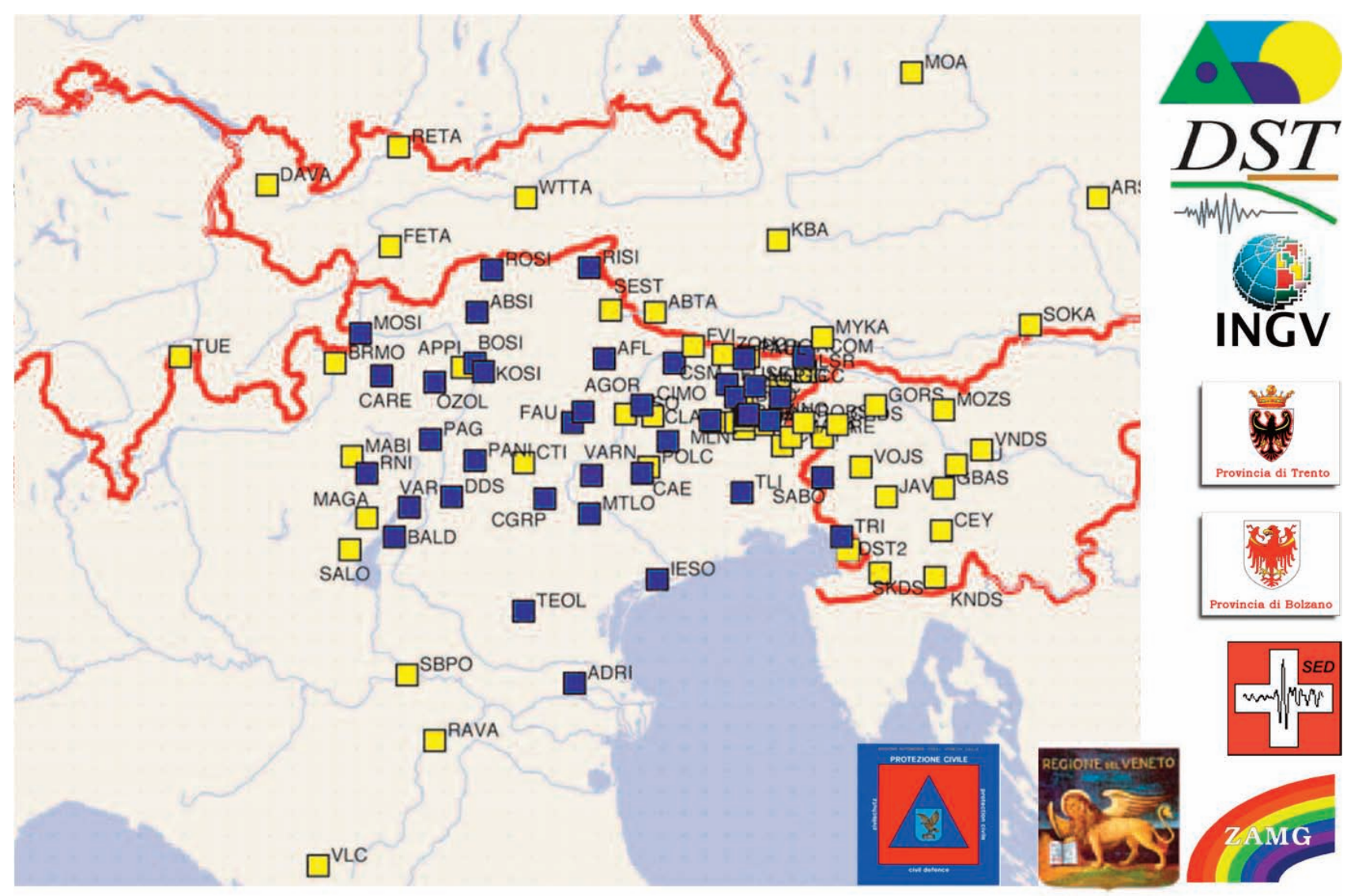

Figure 2. Map of the real-time seismic stations of the OGS-CRS.

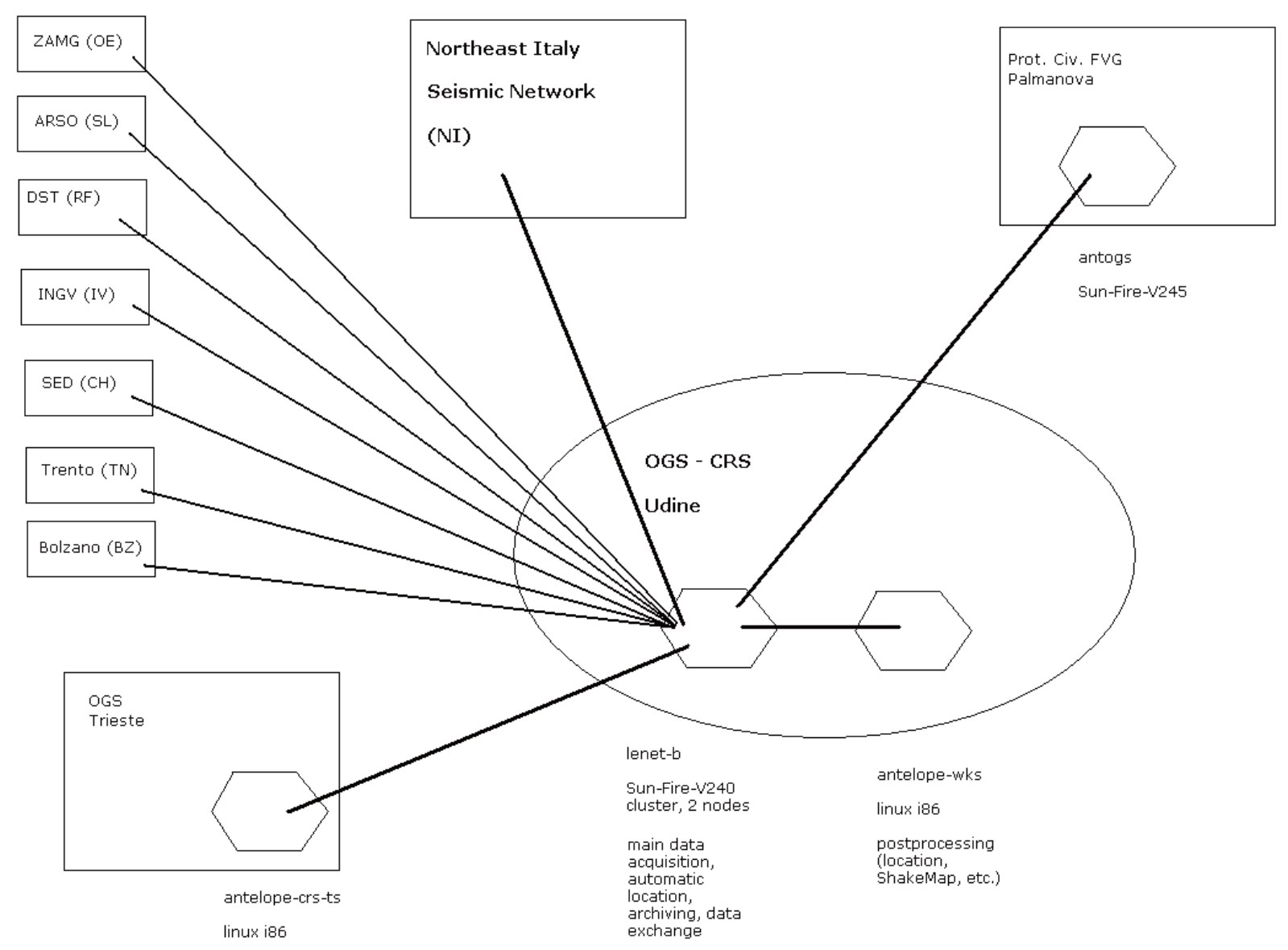

Figure 3. Antelope-based architecture implemented at the OGS-CRS. 


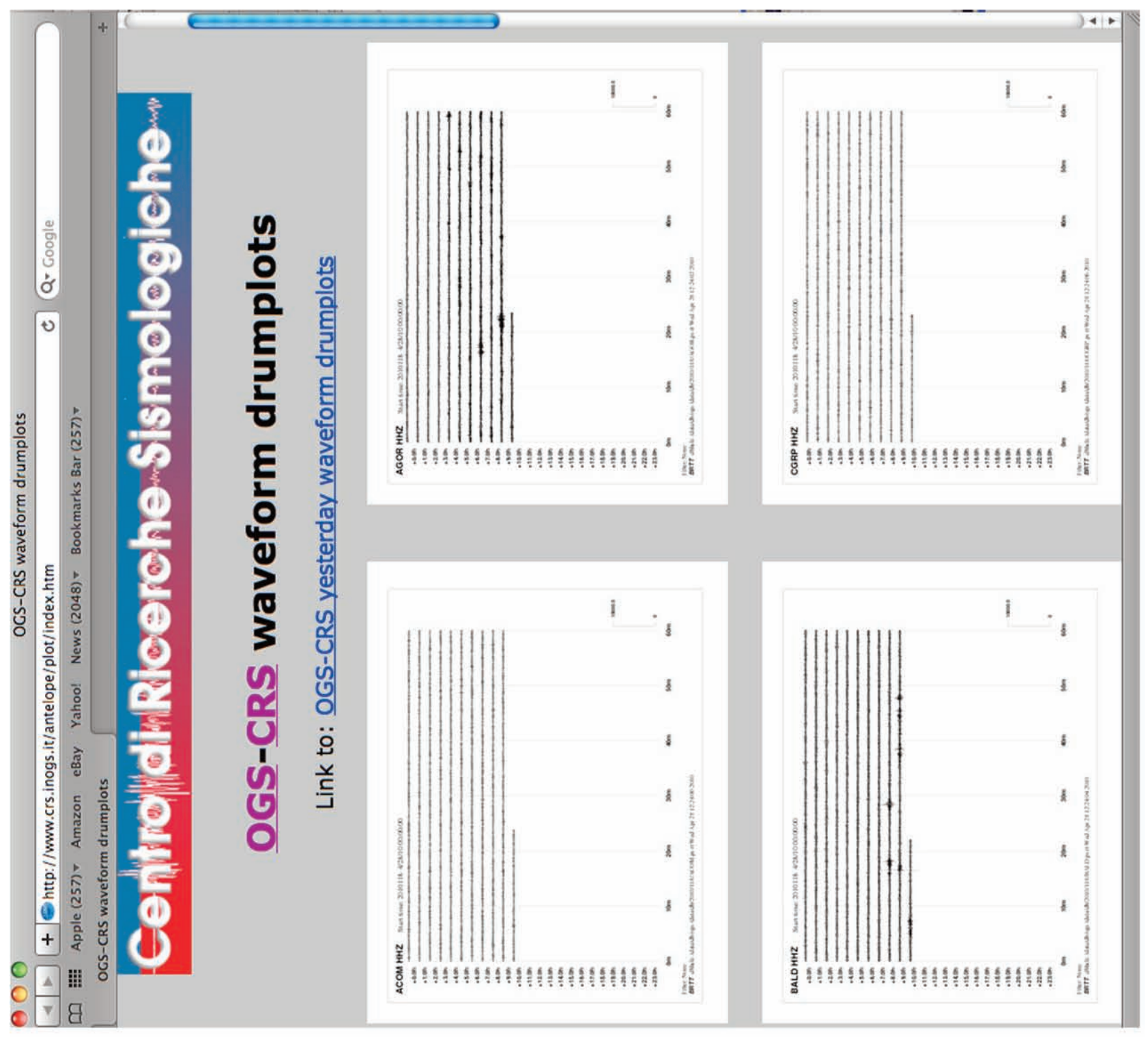

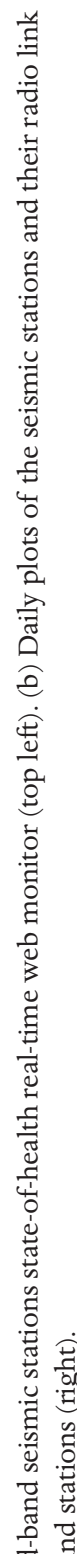
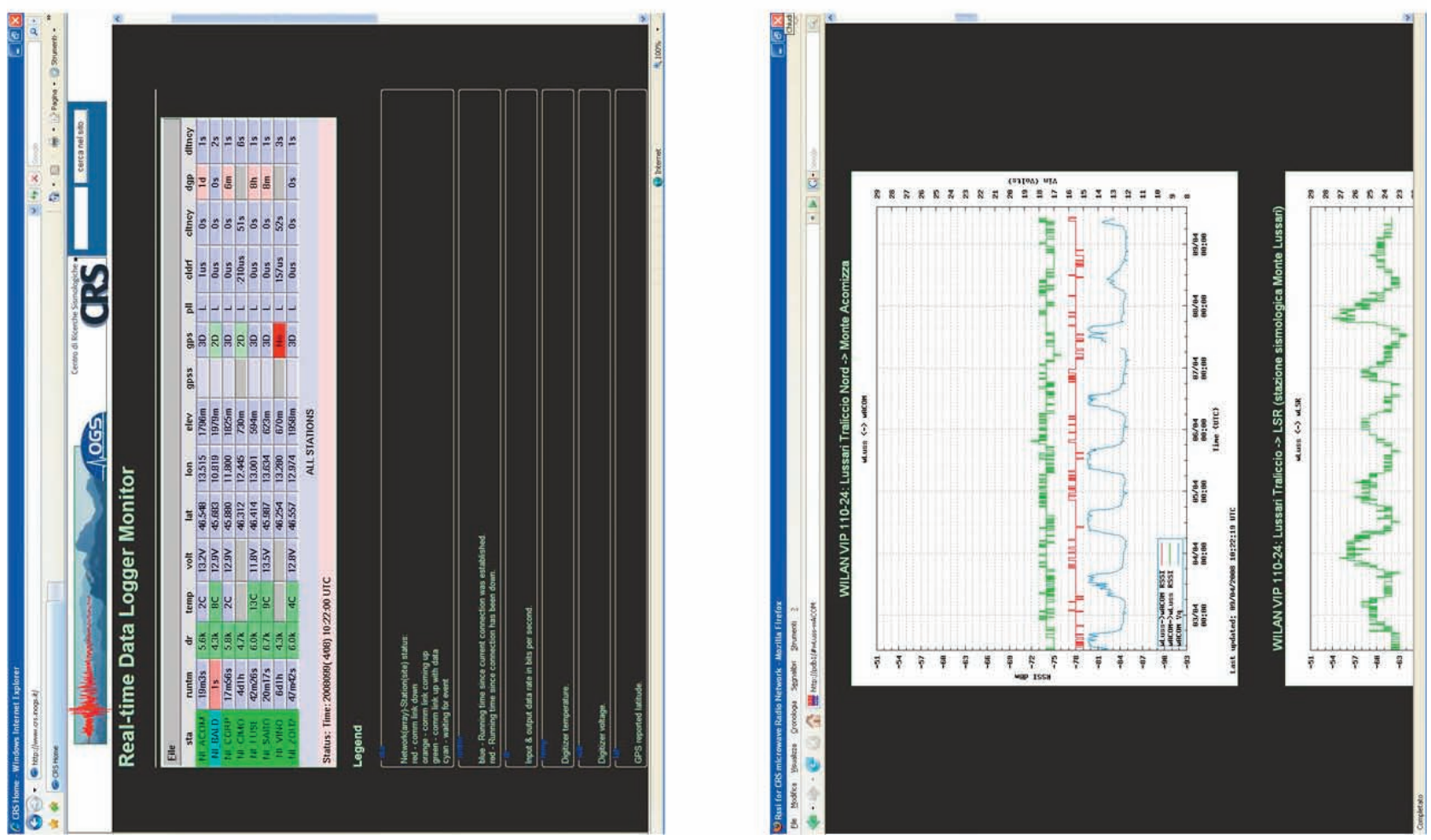

竞 
Data is also forwarded for redundancy to a SUN-Fire-V245 Antelope machine at the Civil Protection Agency of the Friuli-Venezia Giulia region headquarters, and for convenience and redundancy, to a Linux Antelope machine at the OGS-CRS headquarters in Trieste.

Data exchange with other seismological institutions that have adopted the Antelope system (ZAMG, ARSO, University of Trieste, Regione Autonoma Friuli-Venezia Giulia, and Provincia Autonoma di Bolzano) is based on the «orb2orb» protocol. The connection with the other partners is achieved by means of the «seedlink» protocol. The data are imported through internet connections to external SeisComP servers (http:/ / geofon.gfz-potsdam.de / geofon / seiscomp, last accessed on December 3, 2010) using the program «slink2orb» that was developed by Chad Trabant at the Observatories and Research Facilities for European Seismology (ORFEUS). For data export, we maintain a complete SeisComP server that is accessible from the outside and is fed by the Antelope system using a specific plugin («orbplugin»).

The functioning of the network is continuously monitored using the Antelope software tools. State-of-health data are acquired in real time from the Quanterra data loggers, and daily waveform plots are reproduced on dedicated monitors (Figure 4). In addition, we use the popular PASSCAL quick look extended (PQLX) software [McNamara and Boaz 2005] to compute power spectral densities (PSDs) for frequencies ranging from $\sim 0.01 \mathrm{~Hz}$ to $16 \mathrm{~Hz}$ (Figure 5). A comparison of the day and night probability density functions and an examination of artifacts related to station operation and episodic cultural noise allow us to estimate both the overall station quality and the level of Earth noise at each site.

\section{Real-time analysis}

\subsection{Earthquake detection, location and notification}

A set of Antelope programs constitutes the core of the OGS automatic system for earthquake detection and location. Further scripts developed at the OGS-CRS filter earthquakes based on various significance criteria, and then produce and deliver alert messages to the OGS-CRS staff and to the Civil Protection institutions. In particular, single-trace event detection and arrival-time picking is performed running a STA/LTA algorithm on different frequency bands. The Antelope associator puts together the arrival times and locates the earthquakes through a grid search. Alert messages reporting the magnitude and the location of an earthquake are sent within 5 min via fax, e-mail and SMS. Recently, we improved the text of such messages with information about the municipalities where the shaking might be felt by the population or might produce moderate or strong damage. Such preliminary quick and rough estimations should help the Civil Protection authorities to

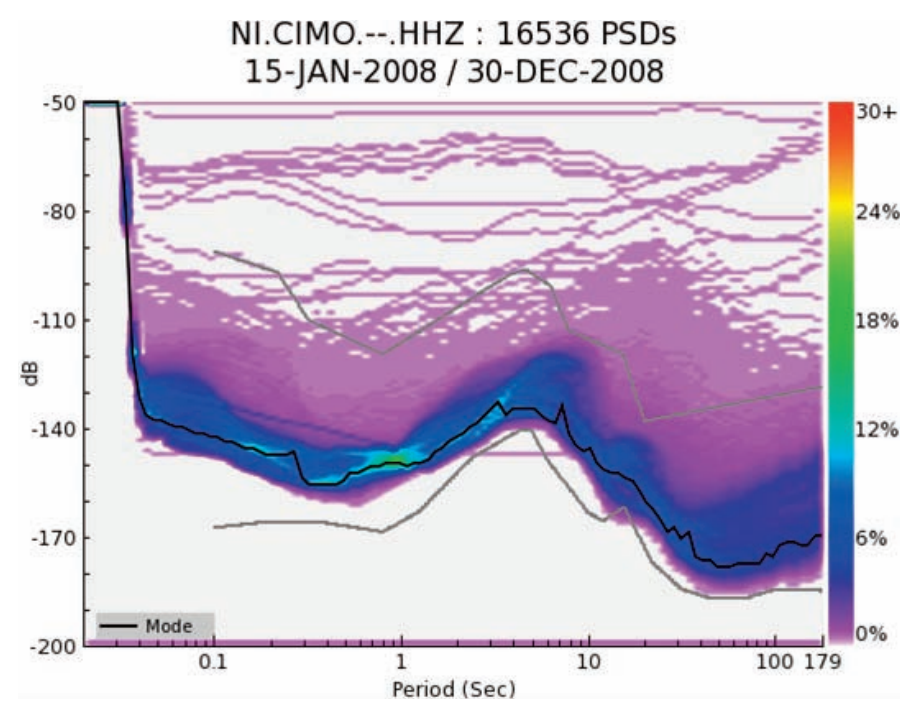

Figure 5. Example of a probability density function of the daily PSDs computed for the HHZ channel of one station of the North-East Italy Seismic Network (CIMO) using one year data (January 15 - December 30, 2008). The gray lines represent the new standard high/low noise model [Peterson 1993], while the black line plots the highest probability power levels.

activate the appropriate level of response. The classification of the municipalities is based on an empirical predictive equation that relates the Mercalli-Cancani-Sieberg (MCS) intensity to the magnitude of the event and the distance from the source. This equation has been calibrated at the OGSCRS for the monitored area. In particular, the equation accounts for the large enhancement of ground motion due to the Moho reflection (up to a factor of three for peak acceleration) that can occur at epicenter distances of between $90 \mathrm{~km}$ and $150 \mathrm{~km}$.

During an emergency (up to $1 \mathrm{~h}$ after an earthquake) and for bulletin compilation purposes, the arrival times and hypocenter locations are manually revised by the CRS analysts. Preliminary checks are performed using the graphical interface of the Antelope program «dbpick», followed by a refined analysis using the "SeisGram2K" software [Lomax 2008]. This program reads the picks and waveforms from the Antelope system through an ad-hoc Java interface that was implemented in our department. The waveforms are analyzed using an internet browser, and the earthquakes are located with HYPO71 [Lee and Lahr 1975]. Some further software has been developed for sending the results back to the Antelope system through an IP connection, to its object ring buffer (orb).

\subsection{Moment tensor computation and shake-map production}

In the framework of a project supported by the Italian Civil Defense Agency and coordinated by INGV (2007-2009 DPC-S3 project), we implemented real-time moment tensor retrieval and shake-map computation. A new website (Figure 6) [Saraò et al. 2009] has also been set-up to display the real-time seismology (RTS) products of the OGS-CRS analyses (the RTS website). 


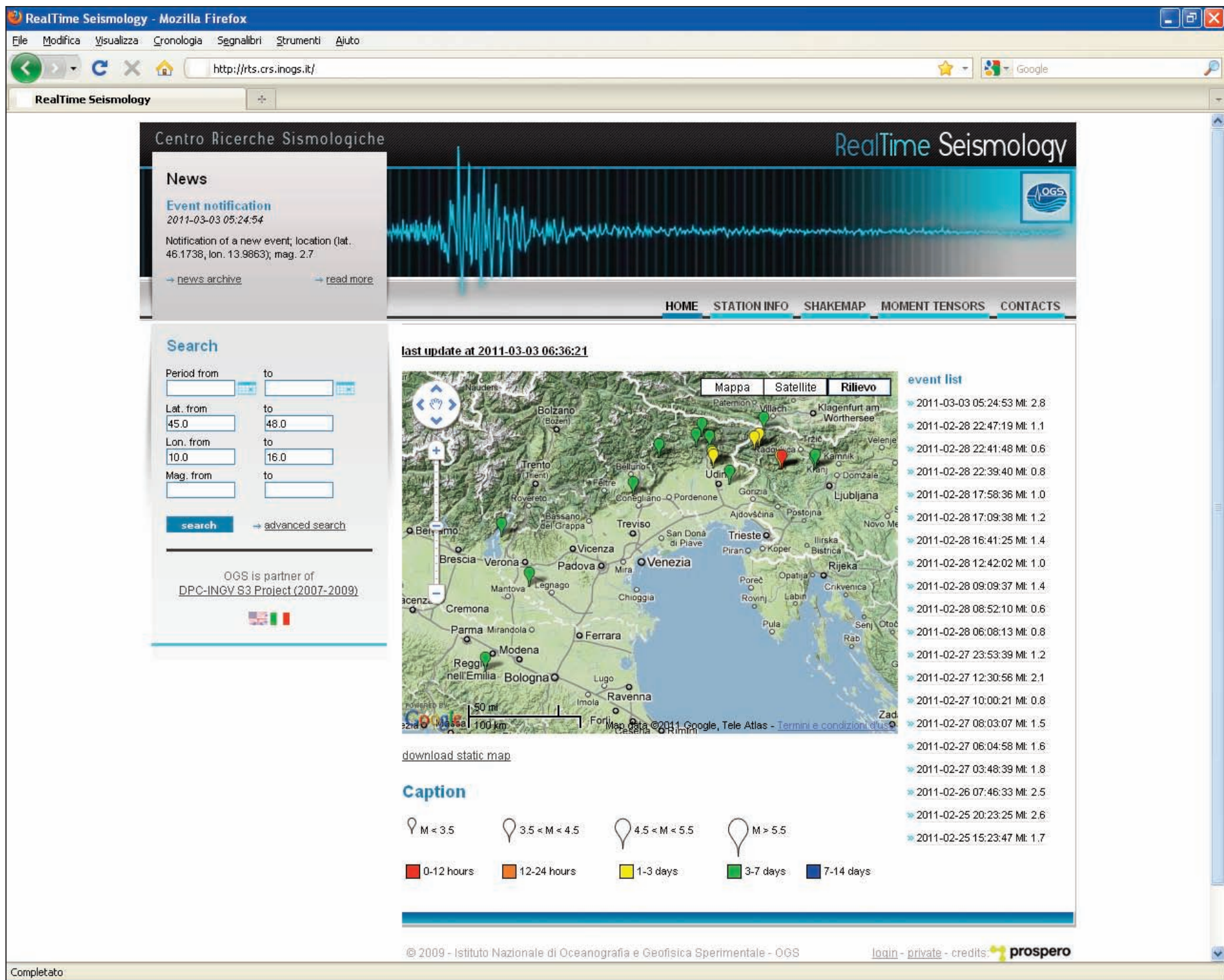

Figure 6. RTS website homepage (http://rts.crs.inogs.it/). The website publishes the map of the seismicity recorded in north-eastern Italy and surroundings, and the shake maps and the moment tensors computed in real time. It also gives information about the OGS-CRS seismic stations.

To compute the moment tensor (Figure 7) by waveform inversion of broad-band waveforms, we implemented, tested and tuned for north-eastern Italy the TDMT_INV code [Dreger 2003], which includes the software of Saikia [1994] for the computation of Green's functions. Several tests were performed using synthetic and real data [Saraò 2007], to check the sensitivity of the solutions according to the OGS broadband network geometry, the number of stations used, and the $1 \mathrm{D}$ velocity models [Bressan 2005] adopted for the area. The feasibility tests revealed that the fault-plane solution and the moment magnitude are quite robust, and that onestation or two-station solutions are effective in many cases [Saraò 2007]. To date, the automatic procedures for momenttensor computation are activated for all of the earthquakes located in north-eastern Italy and its surroundings, starting from $M L=3.7$. Figure 8 shows the plots of some of the moment tensors that have been computed since 2002, using only the data collected at the OGS-CRS.

Since June 2009, the moment tensors have been automatically posted to the RTS website. The moment tensors previously computed [Saraò 2007, Saraò 2008] will be collected in a future publication.

To compute the shaking ground motion produced by $M>2.5$ earthquakes in real time, we installed the ShakeMap software [Wald et al. 2006], as customized for Italy by the INGV [Michelini et al. 2008]. Efforts have been made to ensure its coherency with maps of ground motion computed at other Italian data centers for the same earthquake. At present, the system is fully operative and produces maps of the instrumentally derived intensity, peak ground acceleration and peak ground velocity. For $\mathrm{M}>3$ earthquakes, the system also produces maps for spectral-acceleration responses at 0.3 $\mathrm{s}, 1.0 \mathrm{~s}$ and $3.0 \mathrm{~s}$ (Figure 9). All of the shake maps computed since April 2009 are available on the RTS website.

As is known [e.g. Lin et al. 2005], there can be multiple sources of uncertainty in producing a shake map, due to sparse ground-motion measurements, approximate representations of the fault finiteness and directivity, empirical 
OGS - Centro Ricerche Sismologiche

Tangential

Radial

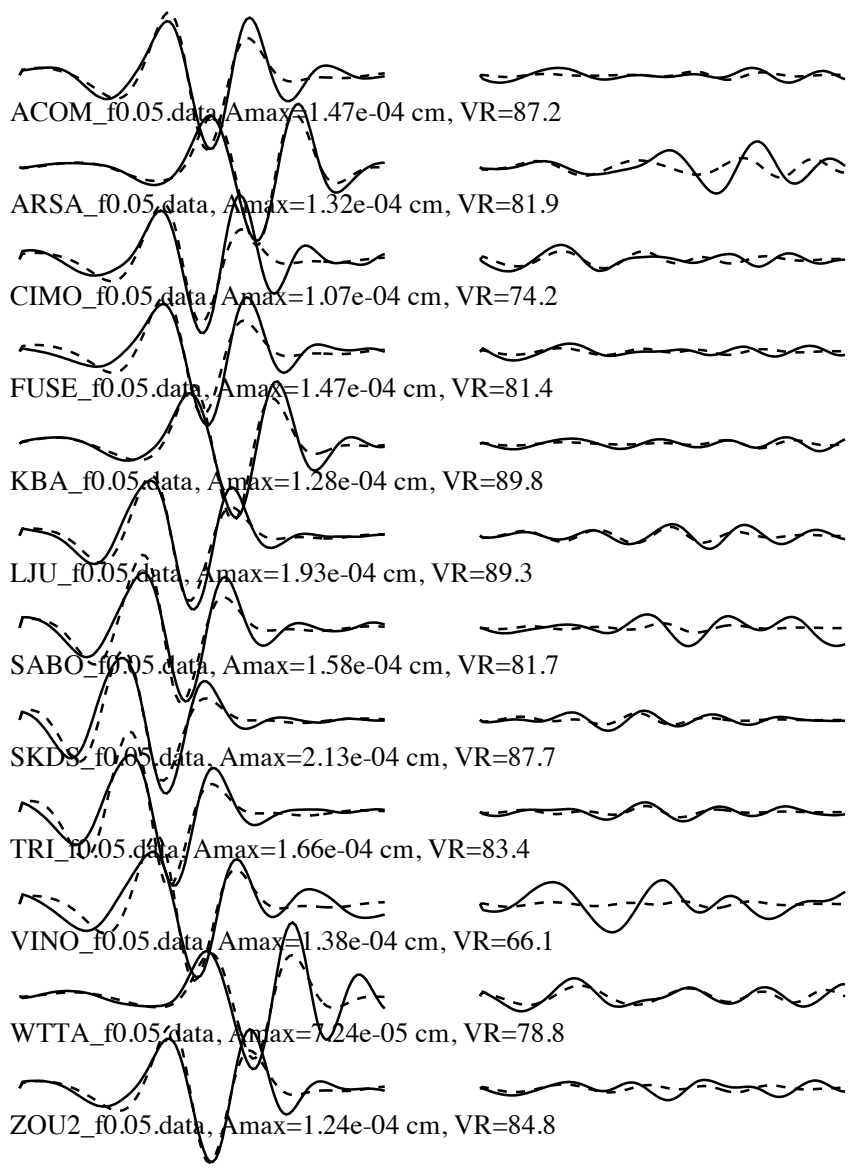

CRS EVENT ID $=17345-18567$

2009/09/20 03:50:15

Depth $=36 \mathrm{~km}$

Strike $=105 ; 14$

Rake $=-171 ;-4$

Dip $=86 ; 81$

Mo $=6.02 \mathrm{e}+22$ dyn $\mathrm{cm}$

$\mathrm{Mw}=4.5$

Percent $\mathrm{DC}=44$

Percent CLVD $=56$

Percent $\mathrm{ISO}=0$ (fixed)

Variance $=1.87 \mathrm{e}-10$

Var. Red $=8.33 \mathrm{e}+01$

$\mathrm{RES} / \mathrm{Pdc} .=4.30 \mathrm{e}-12$
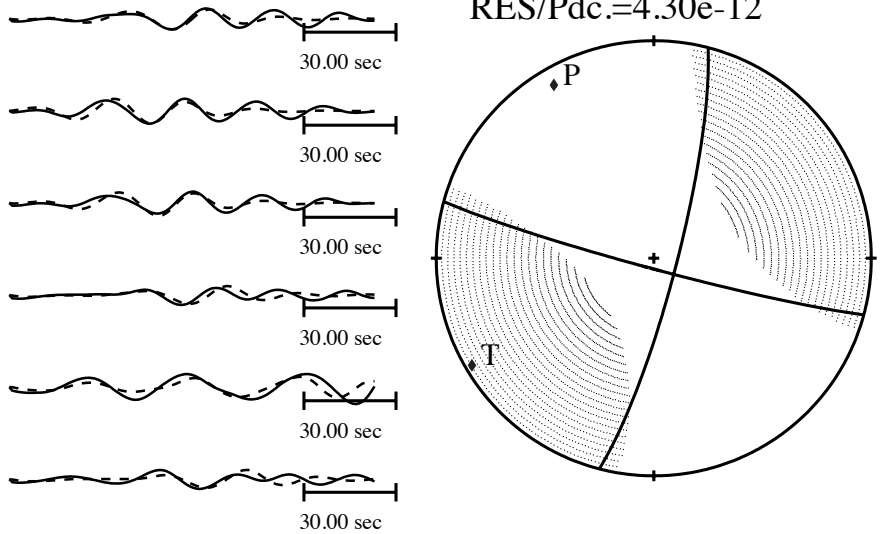

AUTOMATIC SOLUTION

Generated by TDMT_INV code (Dreger, 2001)

Figure 7. Example of a moment tensor retrieved for a $\mathrm{Mw}=4.5$ earthquake using data collected at the OGS-CRS. Left: waveform fit between real (solid line) and synthetics (dashed line). For each trace the code of the station is used, and the maximum amplitude (Amax) in cm and the variance reduction (VR) are reported. Right: the best solution, below the CRS event ID and the date, is given in terms of depth (km), strike, rake, dip, seismic moment (M0), moment magnitude (Mw), percent of double couple (DC), compensated vector linear dipole (CLVD), isotropic (ISO) component, variance, variance reduction (Var. Red) and the variance divided by the DC percentage (RES/PDC). Finally, the plot of the fault plane solution is given (bottom right).

ground-motion predictions, numerical interpolation, and site corrections. To try to reduce some of the uncertainties of the shake maps computed for earthquakes that have occurred in the area monitored by the OGS-CRS, we are carrying out several studies [e.g. Bragato 2009, Moratto and Saraò 2010] related to the improvement of the empirical models specific for the monitored area [Bragato 2009] and the use of rapid synthetics [Moratto and Saraò 2010] that can account for source effects and missing data.

\section{Conclusions}

At the OGS-CRS, we maintain and continuously improve a seismological system that covers the basic requirements like data acquisition, real-time automatic processing, and routine analysis. The main bulk of the system is based on the Antelope software, although the SeisComP software is also used as a real-time data-exchange server tool. Efforts are devoted to maintain an effective exchange of data with other seismological institutions that are active in the area, which increases the detection and location abilities of the system. The latest developments include the real-time moment-tensor computation by waveform inversion and the rapid estimate of groundmotion shaking. For an appropriate ground-motion prediction, we have also developed empirical models that are specific for the monitored area, which account for the large Moho reflection effects observed. A new website has been set-up to report the results of the OGS-CRS RTS analyses. 


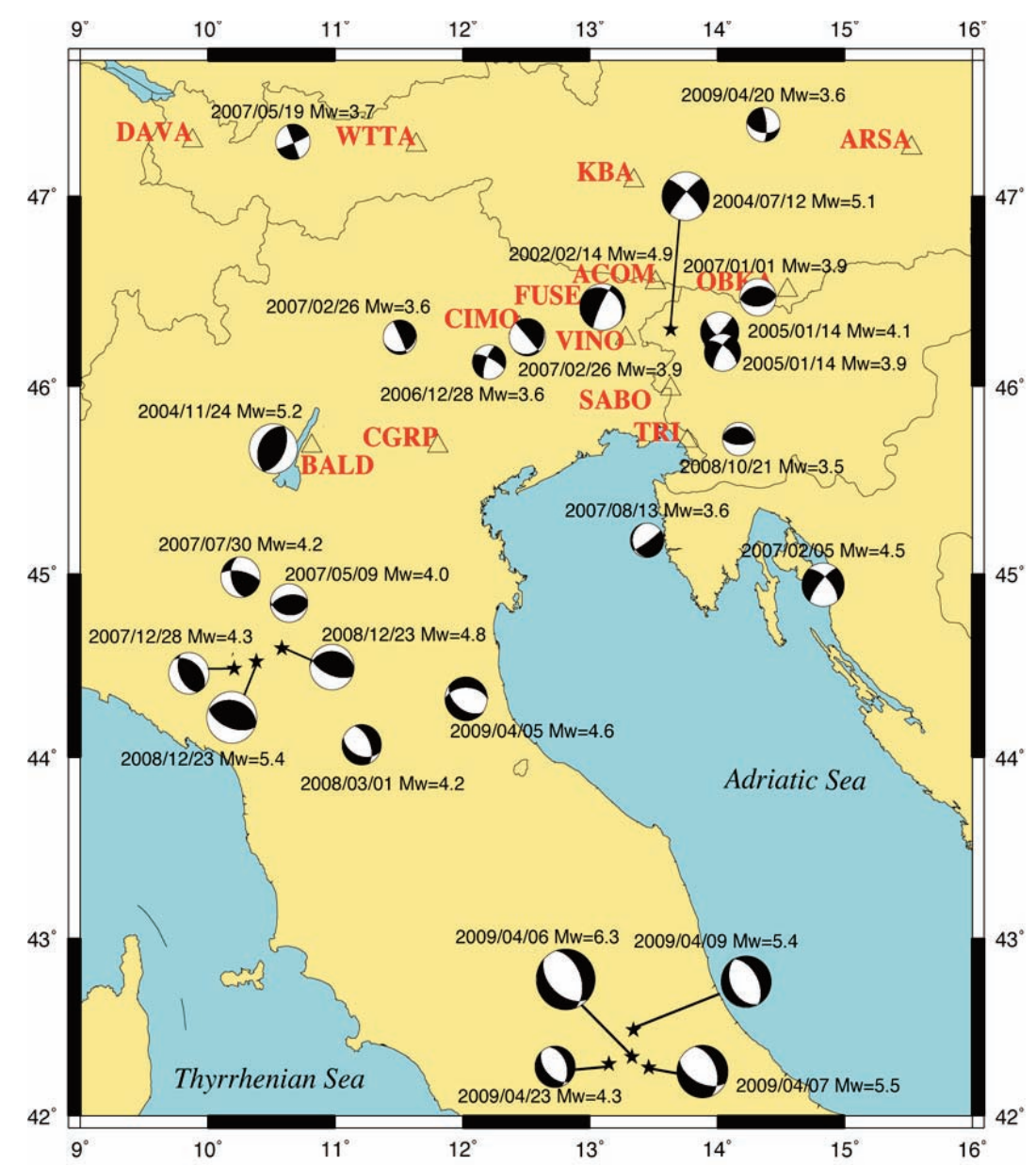

Figure 8. Some of the moment tensor computed using waveforms collected in real-time at the OGS from broad-band seismic stations (triangles). The moment tensors of the 2009 L'Aquila earthquake $(\mathrm{Mw}=6.3)$, together with some of the aftershocks, were computed to test our procedure.

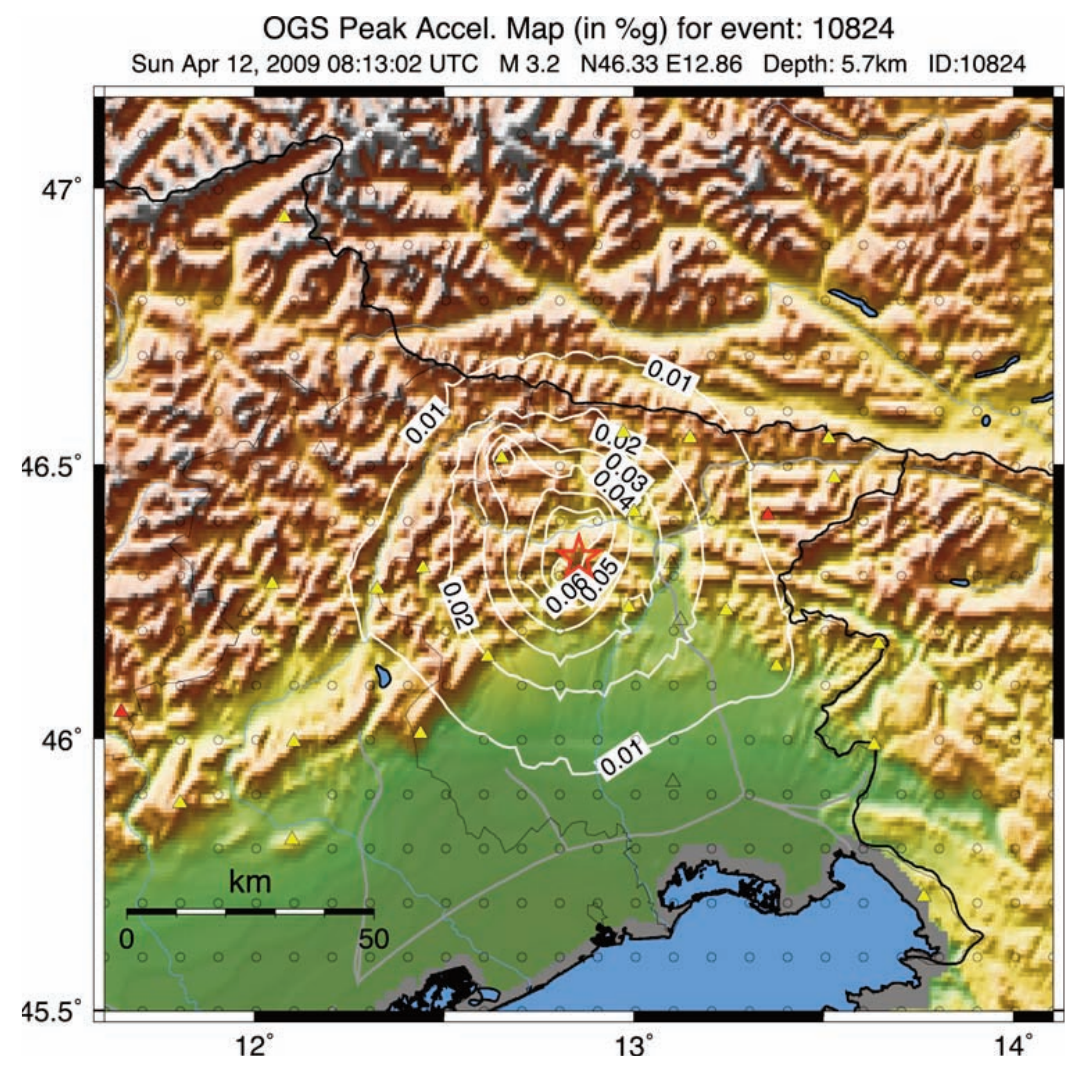

Figure 9. Shake map of the horizontal peak ground acceleration estimated for an ML 3.2 earthquake that occurred in Friuli. 
Acknowledgments. The technical staff of the OGS Seismological Department is acknowledged for its continuous effort in maintaining the North-East Italy Seismic Network. Seismic monitoring in north-eastern Italy is financially supported by Regione Autonoma Friuli-Venezia Giulia and Regione Veneto. The research on moment-tensor computation and shake-map production described in this manuscript has benefited from funding provided by the Italian Presidenza del Consiglio dei Ministri, Dipartimento della Protezione Civile (DPC) under contract 2007-2009 DPC-S3. The GMT software [Wessel and Smith 1991] was used for plotting some of the maps.

\section{References}

Bragato, P.L. (2009). Assessing Regional and Site-Dependent Variability of Ground Motions for ShakeMap Implementation in Italy, B. Seismol. Soc. Am., 99 (5), 2950-2960.

Bressan, G., P.L. Bragato and C. Venturini (2003). Stress and Strain Tensors Based on Focal Mechanisms in the Seismotectonic Framework of the Friuli-Venezia Giulia Region (Northeastern Italy), B. Seismol. Soc. Am., 93 (3), 1280-1297.

Bressan, G. (2005). Modelli di velocità 1D dell'Italia Nord Orientale, Internal report OGS (in Italian) CRS / 5/20/2005, Udine, 18 pp.

Carulli, G.B. and D. Slejko (2005). The 1976 Friuli (NE Italy) earthquake, Giornale di Geologia Applicata, 1, 147-156; doi: 10.1474/ GGA.2005-01.0-15.0015.

Dreger, D.S. (2003). Time-Domain Moment Tensor INVerse Codel (TDMT-INVC) Release 1.1, In: International Handbook of Earthquake and Engineering Seismology, edited by W.H.K. Lee, H. Kanamori, P.C. Jennings and C. Kisslinger, vol. B, 1627.

Lee, W.H.K. and J.C. Lahr (1975). HYP071 (Revised): A computer program for determining hypocenter, magnitude, and first motion pattern of local earthquakes, U. S. Geological Survey Open-File Report 75-311, 113 pp.

Lin, K.W., D.J. Wald, B. Worden and A.F. Shakal (2005). Quantifying CISN Shakemap uncertainties, In: Proceedings of the Eighth U.S. National Conference on Earthquake Engineering, Paper no. 1482.

Lomax, A. (2008). SeisGram2K-Seismogram visualization and analysis software for the Internet-Ver5.3; http: / / alomax.free. $\mathrm{fr} /$ seisgram/SeisGram2K.html (last access, 3 Dec. 2010).

McNamara, D.E. and R.I. Boaz (2005). Seismic Noise Analysis System Using Power Spectral Density Probability Density Functions - A Stand-Alone Software Package, U. S. Geological Survey Open-File Report 2005-1438, 30 pp.

Michelini, A., L. Faenza, V. Lauciani and L. Malagnini (2008). ShakeMap implementation in Italy, Seismol. Res. Lett., 79 (5), 688-697.

Moratto, L. and A. Saraò (2010). Finite source effects in ShakeMap modelled through synthetic seismograms: tests on the $2009 \mathrm{M}_{\mathrm{W}}=6.3$ L'Aquila earthquake, Internal Report OGS 2010/57-CRS/ 9 MODES, Trieste, 22 pp.

Peterson (1993). Observation and modeling of seismic background noise, U. S. Geological Survey Open-File Report
93-322, 94 pp.

Saikia, C.K. (1994). Modified frequency-wavenumber algorithm for regional seismograms using Filon's QuadratureModeling of Lg waves in eastern North America, Geophys. J. Int., 118, 142-158.

Saraò, A. (2007). Seismic moment tensor determination at CRS: feasibility study, Open Report, OGS 2007/60CRS/ 16 MODES, Trieste, 43 pp.

Saraò, A. (2008). Focal mechanisms of NE Italy and surroundings (1928-2008), Open Report OGS 2008/143-CRS / 20 MODES, Trieste, $73 \mathrm{pp}$.

Saraò, A., P.L. Bragato and P. Bernardi (2009). Real-Time Seismology@CRS; http://rts.crs.inogs.it (last accessed, 3 Dec. 2010).

Wald, D.J., C.B. Worden, V. Quitoriano and K.L. Pankow (2006). ShakeMap® Manual. Technical Manual, Users Guide, and Software Guide (Version 1.0, 6/19/06), 156 pp.; http: / / pubs.usgs.gov/tm/2005/12A01/pdf/ 508TM12A1.pdf.

Wessell, P. and W.H.F. Smith (1991). Free software helps map and display data, EOS Trans. AGU, 72 (41), 441.

\footnotetext{
${ }^{\star}$ Corresponding author: Damiano Pesaresi, Istituto Nazionale di Oceanografia e di Geofisica Sperimentale - OGS, Dip. Centro di Ricerche Sismologiche (CRS), Udine, Italy; email: dpesaresi@inogs.it.
}

(C) 2011 by the Istituto Nazionale di Geofisica e Vulcanologia. All rights reserved. 